\title{
USING MUlTiLinear FEATURE SPACE TO ACCELERATE CNN CLASSIFICATION
}

\author{
Michel Andre L .Vinagreiro ${ }^{1}$, Edson C. Kitani ${ }^{2}$, \\ Armando Antonio M. Lagana ${ }^{1}$ and Leopoldo R. Yoshioka ${ }^{1}$ \\ ${ }^{1}$ Laboratory of Integrated Systems,Escola Politecnica da \\ Universidade de Sao Paulo, Sao Paulo, Sao Paulo,Brasil \\ ${ }^{2}$ Department of Automotive Electronics, Fatec Santo Andre, \\ Santo Andre, Sao Paulo, Brasil
}

\begin{abstract}
Computer vision plays a crucial role in ADAS security and navigation, as most systems are based on deep CNN architectures the computational resource to run a CNN algorithm is demanding. Therefore, the methods to speed up computation have become a relevant research issue. Even though several works on acceleration techniques found in the literature have not yet been achieved satisfactory results for embedded real-time system applications. This paper presents an alternative approach based on the Multilinear Feature Space (MFS) method resorting to transfer learning from large CNN architectures. The proposed method uses CNNs to generate feature maps, although it does not work as complexity reduction approach. When the training process ends, the generated maps are used to create vector feature space. We use this new vector space to make projections of any new sample in order to classify them. Our method, named MFS-CNN, uses the transfer learning from pre trained CNN to reduce the classification time of new sample image, with minimal loss in accuracy. Our method uses the VGG-16 model as the base CNN architecture for experiments; however, the method works with any similar CNN model. Using the well-known Vehicle Image Database and the German Traffic Sign Recognition Benchmark we compared the classification time of original VGG-16 model with the MFS-CNN method and our method is, on average, 17 times faster. The fast classification time reduces the computational and memories demand in embedded applications that requires a large CNN architecture.
\end{abstract}

\section{KEYWORDS}

Convolutional Neural Networks, Deep Learning Acceleration, Advanced Driver Assistance Systems.

\section{INTRODUCTION}

The use of computer vision in Advanced Driver-Assistance Systems (ADAS) for environment mapping with images turns possible the recognition of persons, lane road, animals, vehicles, and traffic signs in real-time. The first algorithms designed for computer vision were based on image processing techniques, such as colour segmentation, the histogram of oriented gradients, and cross-correlations. Image processing techniques show good performance for time operation and have an easy implementation. The drawbacks of the techniques above are loss of performance in different light conditions, severe precipitation, mist, and occlusions. In this way, the necessity of robust solutions for ADAS environments rise and, the application of neural networks and Convolutional Neural Networks (CNNs) turns a new research field.

David C. Wyld et al. (Eds): CMC, NCO, SOFT, CDKP, MLT, ICAITA - 2021

pp. 109-122, 2021. CS \& IT - CSCP 2021

DOI: $10.5121 /$ csit.2021.111109 


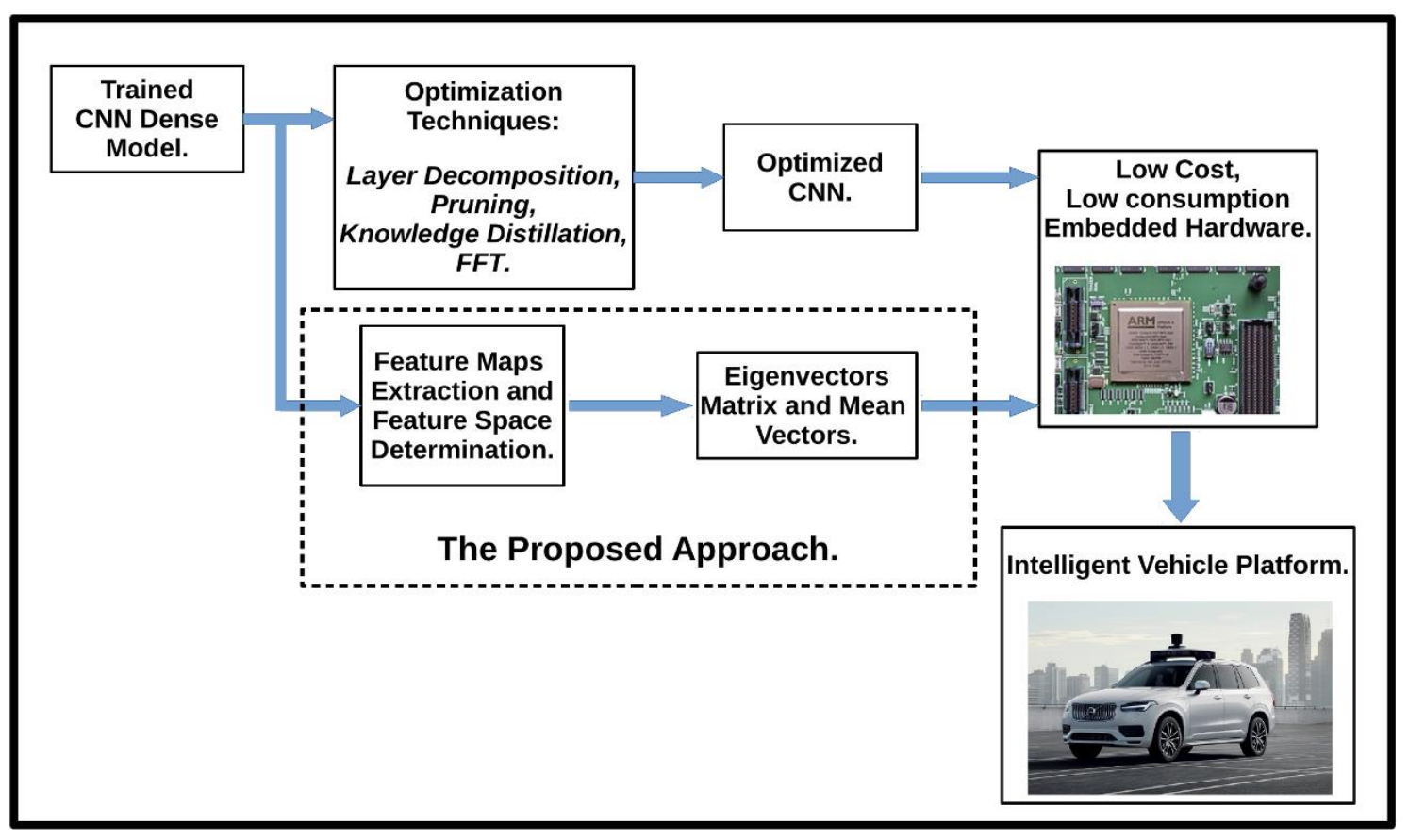

Figure 1. Illustration of the CNN reduction methods for ADAS applications. After train the dense model, the traditional methods reduces the complexity of the model. After the reduction, the embedded hardware platform host the minimal model. Differently, our proposed framework does not reduce the model complexity, however uses the kernel knowledge presents on the maps to determine the feature spaces and use it for the classification process.

At the end of the '90s, Lecunet.al published in [1] and [2] the development and application of Convolutional Neural Networks (CNN). CNN is considered a Deep Learning algorithm and achieved the best performance in image recognition, localization, and segmentation tasks, compared with the traditional image processing techniques [3] and [4], mainly due to CNN's ability to extract a large number of features from input images. When Krizhevsky et al., [5] won the Imagenet-2012 challenge, the breakthrough occurred, achieving a significant performance improvement than previous architecture. Another successful architecture is the deep neural network proposed in [6], called VGG-16 (Visual Geometry Group), which showed the importance of depth architecture to achieve high-performance classification tasks.

Large-scale CNN networks such as VGG-16 is applicable in many classification tasks, including $\mathrm{ADAS}$, mainly used for visual detection and mapping the environment. Computer vision is a essential subsystems that compose ADAS used in vehicles mainly for safety, lane keeping, and collision avoidance systems. CNN is often used in self-driven vehicles to detect and recognize vehicles, persons, animals, and other obstacles. However, CNN's application for real-time operation requires more attention when running in vehicle's embedded platforms due to the need for high-spec hardware (RAM, CPU, and GPU). Some new approaches proposes to deal with the real-time requirements as the problem mentioned above. One is the development of CNN architectures with high performance and low computation cost [7] or compact and less powerful versions of large-scale architectures [8]. Other research lines focus on accelerating the classification time of large CNNs using strategies to optimize kernel activations [9]. The method uses the Single Value Decomposition (SVD) as a low-rank approximation approach to accelerate the classification time of very deep CNNs. Other researches that present methods for acceleration of CNNs are [10] and [11]. In [12], the authors present a study on the relationship between operating speed and accuracy in CNN network's applications used in the object detection within 
an image. That work conducts a study of the balance between accuracy and time of operation through variations of characteristics of the architectures, such as extractors of features, resolution of the input images, etc. The study published in [13] proposes to factorize the convolutions into 2D kernels instead of 3D convolutions. The work reports that the accuracy did not reduce severely and, the time of classification and training decreased a lot. The method proposed in [14] is an evolution of pruning methods for large CNN architectures [15]. The method's purpose is to use the PCA to the network analysis to discover and determine which kernels produce the largest variance results during the training process, thus reducing the accumulated error. Using those kernels and layers, the CNN model is retrained with a compressed version of the architecture. Figure 1 shows the applicability of CNN reductions methods and our proposed framework for the ADAS platform. Unlike the methods presented previously, this paper presents a new approach applied to any large-scale CNN architectures. It uses feature maps for determining the reduced dimensional space. Using this new space, we generate low dimensional samples and train an external classifier. In figure 2 show our proposed method. Despite the universality of our method, we will use the VGG-16 network as the basis for the experiments to validate our method's effectiveness. The rest of the paper is organized as follows. Section II describes a basic CNN structure and an overview of the PCA and MPCA method applied to pattern recognition in images, section III describes the proposed method. Section IV presents the experiments and discusses the results. Finally, section V presents the conclusion.

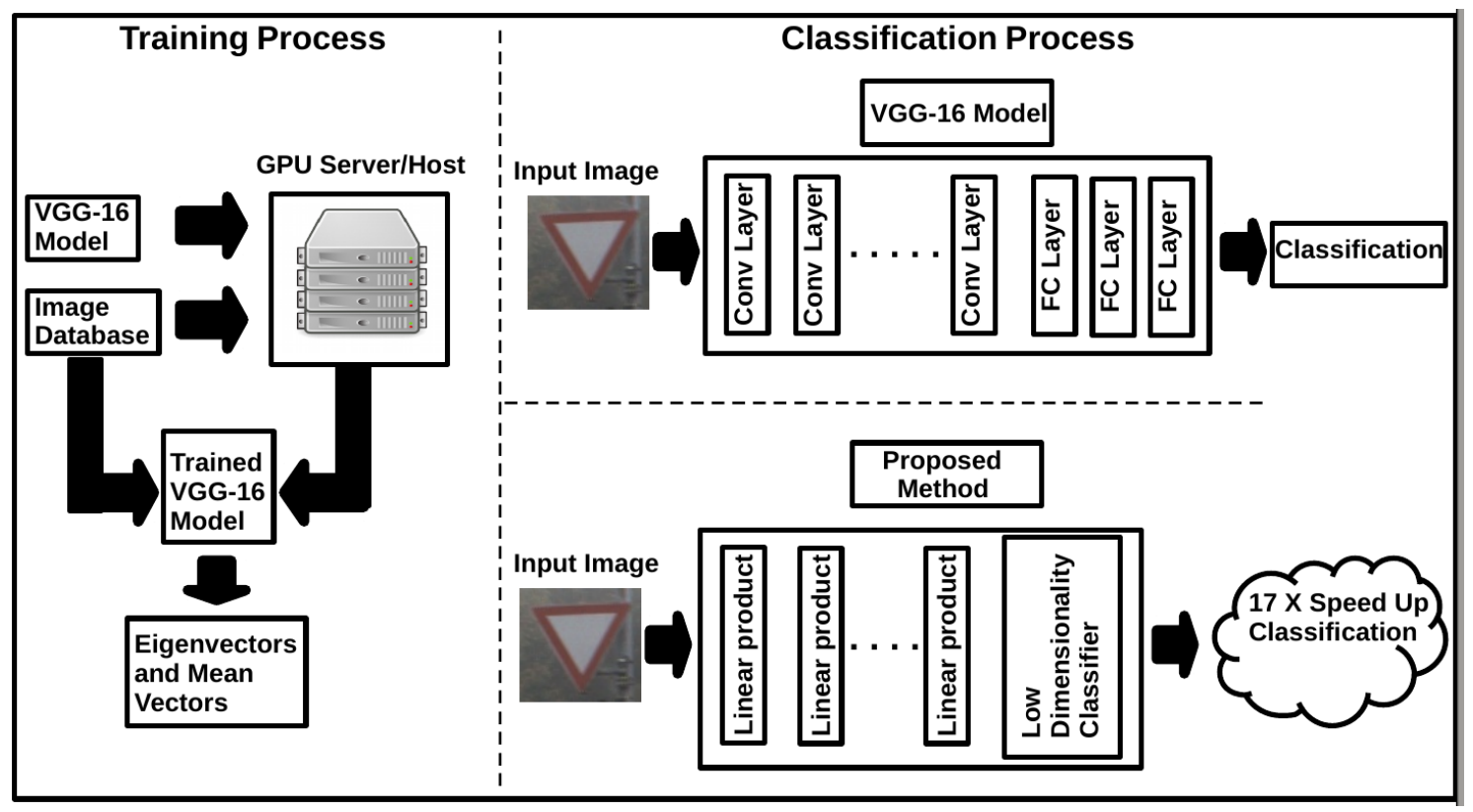

Figure 2. Left side: We train the VGG-16 model on the database with a cloud GPU server. With the trained model, we use the same database to extract banks of eigenvectors and mean vectors used to reduce the architecture. Right side: The VGG-16 model and the proposed method comparison: The substitution of the convolution processes by the chain of products accelerate the classification 17 times.

\section{TheOretical BaSEMEnTS}

\subsection{Convolutional Neural Networks}

CNN structures are usually composed of three types of layers. The first, called the convolutional layer, has the function of extracting many features from images by convolution processes between regions of the input image and the layers' kernels. Every internal kernel element is an 
adjustable parameter adapted during the training phase, and the activation function determines the final output of the kernel [16]. The kernel slides by the whole image according to the parameters stride and padding. All the convolution process outputs are arranged in the feature map matrix [16], and the kernel for each convolutional layer generates the feature maps. The second layer, called sub pooling, uses the feature maps generated by previous convolutional layers. The regions of feature maps are sub-sampling, and the output of the layer is a reduced dimension feature maps. The operators of subsample can be the maxpooling, meanpooling, or minpooling. The maxpooling operator is the most used. Finally, the third layer, called fully connected (FC), consists of neuron units disposed of in interconnected multilayers. The input of the first FC layer consist of all flattens feature maps from the last convolutional layer. The last FC layer can be a probabilistic function or a classifier, such as Support Vector Machines (SVM) or Radial Basis Functions (RBF).

\subsection{PCA and MPCA}

One of PCA's main applications [17] in image processing has been the dimensionality reduction of samples. Even though PCA has 120 years, since Karl Pearson proposed it in 1901, it remains very current and useful. Fundamentally, PCA creates a centred and orthogonal basis from the data's covariance matrix, maximizing the variance corresponding to the largest eigenvalues. This orthogonal basis is used to map the input data X into this new PCA space rotating the data distribution according to the highest variance feature to the lowest nonzero variance feature. Formally, PCA will find an orthogonal matrix $\boldsymbol{\Phi}$ that maps $\mathbb{X} \in \mathbb{R}^{\mathrm{n}}$ to $\mathbb{Z} \in \mathbb{R}^{\mathrm{p}}$, where $\mathrm{p}<<\mathrm{n}$.

The eigenvectors of $\mathbb{X}$ covariance matrix are called Principal Components of set $\mathbb{X}$ new feature space. The projection of any arbitrary $\boldsymbol{x}$ sample into the new PCA feature space can be defined by $\mathbf{z}=\boldsymbol{\Phi}^{\mathrm{T}} \mathbf{x}$, where $\boldsymbol{\Phi}$ is an orthogonal matrix whose the $k t h$ column is the $k t h$ eigenvector from the covariance matrix $\boldsymbol{\Sigma}=\boldsymbol{\Phi} \boldsymbol{\Lambda} \boldsymbol{\Phi}^{\mathrm{T}}$ and $\boldsymbol{\Lambda}$ is the diagonal matrix whose $k$ is the $k$ th eigenvalue of $\boldsymbol{\Sigma}$.

The idea behind the PCA is that the projection of any sample $\boldsymbol{x}$ from the original space to the new PCA space will not change the original distribution once PCA is a linear approach based on the covariance matrix $\boldsymbol{\Sigma}$ of input matrix $\boldsymbol{X}$. However, to deal with tensors in the CNN convolution layer, we need to consider a different approach, such as Multilinear PCA (MPCA) as proposed by [18].

Lu et al. [18] proposed Multilinear PCA (MPCA) for tensor objects as a multidimensional object, mainly related to videos and images. Considering a sequence of frames from a video file, $\mathbf{A} \in$ $\mathbb{R}^{11 \times 12 \times \ldots \times 1 n}$ will be the tensor object of nth-order and each frame $\mathbb{U}_{\mathrm{lk}} \in \mathbb{R}^{\mathrm{i} \times \mathrm{j}}$, where $\mathrm{k}=1,2, \ldots, \mathrm{N}$. Although, MPCA will reduce the total dimensionality from $\mathrm{N} \times \mathrm{i} \times \mathrm{j}$ to $\mathrm{P} \times \mathrm{i} \times \mathrm{j}$, where $\mathrm{P}<<\mathrm{N}$.

The MPCA requires a stack of input data $\mathbf{X}_{\mathrm{k}} \in \mathrm{R}^{\mathrm{i} \times \mathrm{j}}$ to project the tensor object $\mathbf{A}$ to the new reduced tensor space. The reduction occurs by the product of tensor $\mathbf{A}$ by a matrix $\mathbf{U} \in \mathrm{R}^{\mathrm{in} \times j \mathrm{j}}$ denoted as $\mathbf{A} \times \mathbf{U}$, and $\mathbf{U}$ corresponds to the $\mathrm{N}$ projections matrices that maximize the $\mathrm{M}$ scatter of the tensors defined by $\boldsymbol{\psi}_{\mathrm{A}}=\mathbf{\Sigma}_{\mathrm{M}}\left\|\mathbf{A}-\mathbf{A}_{\mathbf{m}}\right\|$, where $\mathbf{A}_{\mathbf{m}}$ is the mean tensor.

\section{The Proposed Method}

\subsection{Definition}

The proposed method adapted for VGG-16 is divided into four phases, as shown in the following:

- Phase 1: Initial step consists of applying the pre-processing to convert all images to the grayscale and resize them to $224 \times 224$ pixels. 
- Phase 2: The original VGG-16 model is trained with these pre-processed samples.

- Phase 3: $M$ image samples of the training subset, with $M<N$, are presented to the trained VGG-16 model and generates $K_{l}$ feature maps for each image in each layer $l=\{1,2,3, \ldots, 13\}$ were $K_{l}$ is the number of kernels of the layer $l$. Each feature map is concatenated and arranged in the matrix $\boldsymbol{X}^{(l)}$ of size $V \times n$, where $V$ is the product of $M$ per $K_{l}$ and, $n=H^{2}$, where $H \times H$ is the input size. Before applying the PCA, the mean vector of $\boldsymbol{X}^{(l)}$ is extracted and stored:

$$
\boldsymbol{x}_{m}^{(l)}=\frac{1}{V} \sum_{i=1}^{V} \boldsymbol{x}_{i}
$$

The covariance matrix of $\boldsymbol{X}^{(l)}$ is computed as:

$$
\operatorname{Cov}\left(\boldsymbol{x}^{(l)}\right)=\frac{1}{V} \sum_{i=1}^{V}\left(\boldsymbol{x}_{i}-\boldsymbol{x}_{m}^{(l)}\right)\left(\boldsymbol{x}_{i}-\boldsymbol{x}_{m}^{(l)}\right)^{T}
$$

The $p_{l}$ eigenvectors of the covariance matrix of $\boldsymbol{X}^{(l)}$ related with nonzero eigenvalues compose the matrix $\boldsymbol{A}^{(l)}$, with dimensionality $p_{l} \times n$. The matrix $\boldsymbol{A}^{(l)}$ and the mean vector $\boldsymbol{x}^{(l)}$ are the output of phase three.

- Phase 4: The last step consist of applying phase three for all layers of the model.

For each layer $l$, the feature maps must be resized to $H \times H$, where $H=\sqrt{ } p_{(l-1)}$, except for the first layer. This resize turns possible the process of the dot product that will generate the low dimensional samples.

At the end of all phases, matrices of eigenvectors and mean vectors for all layers are generated. In the dense models, the chain of subtractions and products using the matrices of eigenvectors and the mean vectors replaces convolutional processes. This replacement accelerates the time of classification.

The main objective of this work is to reduce the overall classification time for a new image sample. We call our proposed method Multilinear Feature Space Convolutional Neural Network (MFS-CNN).

Figure 3 illustrates the proposed method. 


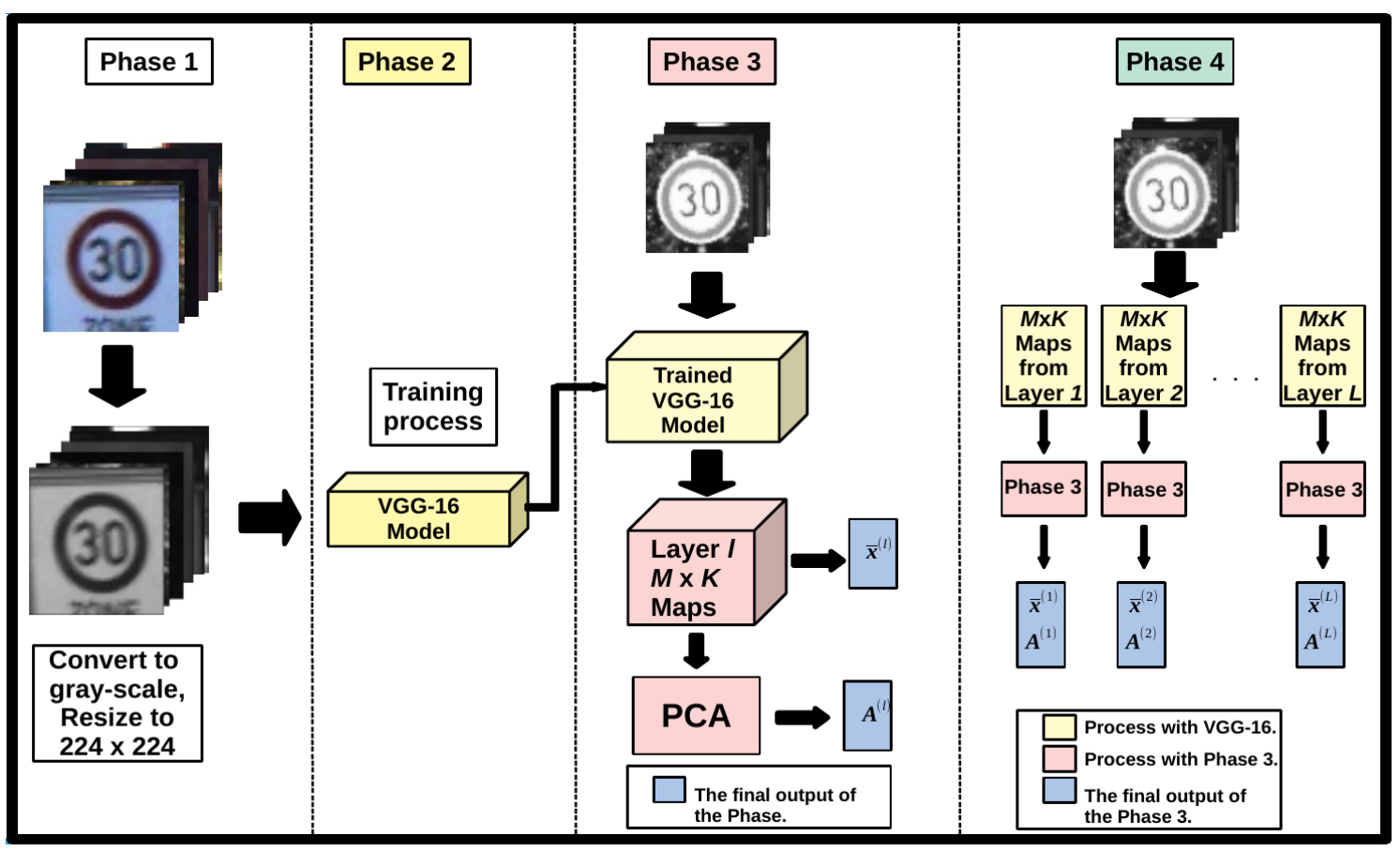

Figure 3. The illustration of the processes to obtain the matrix of eigenvectors and the mean vector for each layer. In phase one, all samples of the image database are pre-processed. The image samples are converters to grey-scale and resize to $224 \times 224$. After the pre-processes, phase two consists of the training and validation of the VGG-16 model. In phase three, $M$ image samples are presented to trained VGG-16 model to generate $M \times K_{l}$ features maps per layer. For each $l$ layer, the mean vector $\boldsymbol{x}^{(l)}$ and the matrix of eigenvectors $\boldsymbol{A}^{(l)}$ are computed. Phase 4 consists of computing the mean vector and the eigenvectors matrix for all layers of the original VGG-16 model.

\subsection{Generating Samples with Low Dimensionality}

Projecting any new image onto Feature Map Space requires resizing the image sample, Imt, in $224 \times 224$ pixels. In the second step, the new image is concatenated to vector $x t, 1 \times n$, with $n=$ 50176. The projection of $x t$ into space of the first layer, $z(x t)^{(1)}, 1 \times p_{1}$ occurs by the subtraction of mean vector $\boldsymbol{x}^{(1)}$ and the dot product with $\boldsymbol{A}^{(1)}$. The vector $\boldsymbol{z}(\boldsymbol{x t})^{(1)}$ is projected into space of the second layer by the same process above, generating $z(x t)^{(2)}$, and then projected into space of the third layer, and repeating the process until the last layer as shown in equations 3.3, 3.4 and 3.5 respectively.

$$
\begin{gathered}
\boldsymbol{z}(\boldsymbol{x} \boldsymbol{t})^{(1)}=\left(\boldsymbol{x} \boldsymbol{t}-\boldsymbol{x}_{m}^{(1)}\right)\left(\boldsymbol{A}^{(1)}\right)^{(T)} \\
\boldsymbol{z}(\boldsymbol{x} \boldsymbol{t})^{(2)}=\left(\boldsymbol{z}(\boldsymbol{x} \boldsymbol{t})^{(1)}-\boldsymbol{x}_{m}^{(2)}\right)\left(\boldsymbol{A}^{(2)}\right)^{(T)} \\
\boldsymbol{z}(\boldsymbol{x} \boldsymbol{t})^{(L)}=\left(\boldsymbol{z}(\boldsymbol{x} \boldsymbol{t})^{(L-1)}-\boldsymbol{x}_{m}^{(L)}\right)\left(\boldsymbol{A}^{(L)}\right)^{(T)}
\end{gathered}
$$

As mentioned early, the low dimensional samples are used to train and validate an external classifier that substitutes the fully connected layers of the VGG-16 model. 


\section{EXPERIMENTAL RESUlts}

A set of experiments were conducted to evaluate the capability of MFS-CNN to speed up the classification time with minimal accuracy loss. The first experiments with six scenarios varying parameters were conducted. To exploit the best scenario, we used the cross-validation experiments at all scenarios and, the results were organized and presented in tables. To prevent overfitting, the training and validation of all classifiers use the early stopping method.

The difficulty of reproduction of CNN's reduction approaches turns impractical the use in experiments with these approaches. These implementations are crucial for compare our proposed method and obtain an overall situation of the proposed method in the research area.

\subsection{Datasets}

\subsubsection{Vehicle Image Database}

Universidad Politécnica de Madrid [19] to evaluate computer vision algorithms for automotive applications built it. This image database is composed of 7325 images of road lanes with the presence of vehicles or not. The images belonged to two classes and were collected under a different angle, environments, and light conditions. The images have dimensions of $64 \times 64$ pixels. Because of the unbalance of the database, with a different number of images per class and corrupted images, we used only 5400 of 7325 available. The best result achieved in the training process of model VGG-16 was $98.7 \%$ accuracy in the test set after 31 epochs, a learning rate of $10^{-6}$, and a mini-batch size of 20.

\subsubsection{German Traffic Sign Detection Benchmark (GTSDB)}

This image database is available at Institut Für Neuroinformatik of Ruhr-Universitat Bochum [20]. The database contains more than 50,000 images of traffic signs distributed in 43 classes. Simultaneously, the images were captured in several environments, different angles of view, light conditions, and different dimensions. We randomly select four classes of images to conduct the experiments. The best result achieved in the training process of the VGG-16 model was $99.7 \%$ of accuracy in the test set at 24 epochs, with learning a rate of $10^{-6}$ and a mini-batch size of 100 .

\subsection{Experiments Scenario Description}

The experiments consist of the training and test of an external classifier with the samples projected on layers. The speedup (SPU) of the time for classification is measured by:

$$
S P U=\frac{t_{V G G}}{t_{M F S_{-} C N N}}
$$

Where $t_{V G G}$ is the time of classification of an arbitrary sample by trained VGG-16 model and $t_{M F S_{-} C N N}$ is the time of classification by our proposed method.

Before initializing the experiments, we have to compute the low dimensional samples considering the first seven and, after this, all layers spaces. For the first layer, were extracted $V-1$ eigenvectors and, for remains layers $p_{(l-1)}$. To compose the matrices of eigenvectors it was used different numbers of eigenvectors $p_{l}$ for each layer. The first ranked eigenvectors chosen from each layer that produced the best result were: 6889, 6724, 4096, 3364, 2304, 2116, 1600, 1444, $1156,1024,900,784,676$, from the first to the last layer, respectively. We used different 
scenarios to conduct the experiments. The experiments use all or part layer spaces to obtain the final vector. Besides, different combinations of eigenvectors to compose eigenvectors' matrix, using these vectors to train and validate the external classifier. In the following, we describe the scenarios used in the experiment.

The scenarios are summarized in table I and the results of each scenario are presented in tables IV to IX, respectively. Before starting the experiments to check if the method effectively speeds up the classification time, we conducted cross-validation experiments to define which classifiers achieve higher accuracy values.

Table 1. Experiments Scenario Description

\begin{tabular}{|c|c|c|}
\hline Scenario & Layers Selected & Eigenvectors Selection \\
\hline 1 & All 13 layers & First Ranked \\
\hline 2 & First seven layers & First Ranked \\
\hline 3 & All 13 layers & Last Ranked \\
\hline 4 & All 13 layers & Randomly \\
\hline 5 & First seven layers & Last Ranked \\
\hline 6 & First seven layers & Randomly \\
\hline
\end{tabular}

\subsubsection{Cross-Validation Experiments}

Before the random selection for subsets mounting, we set the k parameter of the k-fold algorithm as five, which always reserves $20 \%$ of total samples to test.

For each k-fold round, the VGG-16 model is trained with $k-1$ subsets designed for the training process and validate with remain. We used $M=1000$ randomly selected samples from the training subsets for generating the feature maps. All samples of the training subsets generate the low dimensional samples to train the external classifier. The proposed method was validated with the low dimensional samples generated with the same image sample subset used to validate the original VGG-16 model.

In the experiments described in this section were used the following external classifiers: Adaboost, Decision Tree, K-Nearest Neighbour, Naive Bayes, Random Forest and Multi-Layer Perceptron and SVM.

The best cross-validation results were achieved considering scenario 1 . The tables 2 and 3 presents the best results from each classifier using validation subsets.

In the database 1 [19], the best value achieved by the Adaboost classifier occurred when the number of estimators was set to 200. In the KNN classifier, the best value for the $k$ parameter for all folds was 1 . The multi-layer perceptron classifier has three layers. The first layer has 1024 units; the intermediary layer has 256 and, the output layer 2 units. The activation function for the hidden layers and the output layer are Relu and softmax, respectively. The learning rate was fixed in $10^{-4}$, and the mini-batch size was 20 . The best accuracy value in the fold was achieved after 24 epochs. The SVM classifier utilized the linear kernel. 
Table 2. Cross-Validation Accuracy Performance on Database 1, best results highlighted in bold.

\begin{tabular}{|c|c|c|c|c|c|}
\hline Classifier & Fold 1 & Fold 2 & Fold 3 & Fold 4 & Fold 5 \\
\hline Adaboost & $93.0 \%$ & $92.2 \%$ & $91.9 \%$ & $92.9 \%$ & $92.1 \%$ \\
\hline D. Tree & $85.6 \%$ & $83.1 \%$ & $84.6 \%$ & $84.4 \%$ & $84.4 \%$ \\
\hline K-NN & $90.0 \%$ & $88.1 \%$ & $89.4 \%$ & $90.3 \%$ & $89.5 \%$ \\
\hline MLP & $97.1 \%$ & $97.2 \%$ & $96.8 \%$ & $97.0 \%$ & $\mathbf{9 7 . 3 \%}$ \\
\hline N. Bayes & $85.1 \%$ & $83.1 \%$ & $82.0 \%$ & $83.9 \%$ & $82.6 \%$ \\
\hline R. Forest & $86.1 \%$ & $86.9 \%$ & $88.8 \%$ & $88.4 \%$ & $87.8 \%$ \\
\hline SVM & $88.8 \%$ & $88.4 \%$ & $77.5 \%$ & $83.0 \%$ & $89.0 \%$ \\
\hline VGG-16 & $97.8 \%$ & $98.4 \%$ & $97.5 \%$ & $97.6 \%$ & $\mathbf{9 8 . 8 \%}$ \\
\hline
\end{tabular}

In the database 2 [20], due to a large amount of memory required to store eigenvectors' matrices, we randomly choose four classes of 43 . The best value achieved by the Adaboost classifier occurred when the number of estimators was set as 200 . In the KNN classifier, the best value for the $k$ parameter for all folds is 1 . The multilayer perceptron classifier has three layers. The first layer has 1024 units, the intermediary layer has 1024 and, the output layer four units. We used as activation function for the hidden layers and the output layer are Relu and softmax, respectively. The learning rate has fixed in $10^{-5}$, and the mini-batch size is 25 . The best accuracy value in fold 1 occurred at 26 epochs. The SVM classifier utilized the Radial Basis Function kernel.

Table 3. Cross-Validation Accuracy Performance on Database 2, best results highlighted in bold.

\begin{tabular}{|c|c|c|c|c|c|}
\hline Classifier & Fold 1 & Fold 2 & Fold 3 & Fold 4 & Fold 5 \\
\hline Adaboost & $93.6 \%$ & $93.7 \%$ & $93.2 \%$ & $92.2 \%$ & $91.8 \%$ \\
\hline D.Tree & $87.6 \%$ & $88.3 \%$ & $89.1 \%$ & $87.5 \%$ & $88.5 \%$ \\
\hline K-NN & $97.2 \%$ & $97.2 \%$ & $97.9 \%$ & $98.1 \%$ & $97.7 \%$ \\
\hline MLP & $\mathbf{9 9 . 6 \%}$ & $99.2 \%$ & $99.2 \%$ & $99.5 \%$ & $99.2 \%$ \\
\hline N. Bayes & $68.7 \%$ & $69.7 \%$ & $69.4 \%$ & $67.0 \%$ & $68.4 \%$ \\
\hline R. Forest & $83.1 \%$ & $83.2 \%$ & $84.3 \%$ & $84.0 \%$ & $83.7 \%$ \\
\hline SVM & $97.5 \%$ & $98.0 \%$ & $97.7 \%$ & $98.2 \%$ & $97.8 \%$ \\
\hline VGG-16 & $\mathbf{9 9 . 7 \%}$ & $98.5 \%$ & $98.7 \%$ & $99.4 \%$ & $99.2 \%$ \\
\hline
\end{tabular}

Comparing the results presented in the tables, the classifiers that achieved the best overall results were the MLP and SVM, except for the first image database were Adaboost overcome SVM.

\subsubsection{Speedup Experiments}

The accuracy values achieved in the scenario 1 using all databases are closest to the original VGG model. Table 4 summarizes the results achieved in image databases 1 and 2.

Table 4. Speedup Performance on Database 1 and 2 for Scenario 1, best results highlighted in bold.

\begin{tabular}{|c|c|c|}
\hline \multicolumn{3}{|c|}{ Database 1} \\
\hline Classifier & Accuracy & SPU \\
\hline MFS-CNN-MLP & $97.3 \%$ & 16.9 \\
\hline MFS-CNN-Adaboost & $93.0 \%$ & 17.1 \\
\hline VGG-16 & 98.8\% & - \\
\hline \multicolumn{3}{|c|}{ Database 2} \\
\hline Classifier & Accuracy & SPU \\
\hline MFS-CNN-MLP & $99.6 \%$ & 16.8 \\
\hline MFS-CNN-SVM & $98.2 \%$ & 16.8 \\
\hline VGG-16 & $99.7 \%$ & - \\
\hline
\end{tabular}


As expected in scenario 1, the loss compared with VGG-16 is minimal. The minimal loss probably occurs by the use of ordered high representation eigenvectors. That produce high information integrity as related by various works that use the PCA method. However, the best performance of the Adaboost classifier overcome SVM will investigate further.

We can easily conclude that the acceleration compared with scenario number 1 is due to the reduced number of layers. The global augment of loss can suggest that the performance is related to the totality of layers used in the classification task. Despite scenario 3 use all layers, the selection of eigenvectors with less associated eigenvalues decreases the global performance. The use of a random selection of eigenvectors in scenario 4 reduces performance smoothly, but both the accuracy and acceleration remain close to scenario 1 . This minimal loss and high acceleration can indicate high redundancy of eigenvectors.

We can observe that the selection of eigenvectors is irrelevant when the method uses only the first layers. However, we can conclude that the complete solution for architecture reduction uses all layer spaces. Although, understand the operation in the first layers may elevate the acceleration without increase the loss.

Table 5. Speedup Performance on Database 1 and 2 for Scenario 2, best results highlighted in bold.

\begin{tabular}{|c|c|c|}
\hline \multicolumn{3}{|c|}{ Database 1 } \\
\hline Classifier & Accuracy & SPU \\
\hline MFS-CNN-MLP & $95.1 \%$ & 17.3 \\
\hline MFS-CNN-SVM & $83.6 \%$ & 17.5 \\
\hline VGG-16 & $\mathbf{9 8 . 8 \%}$ & - \\
\hline \multicolumn{3}{|c|}{ Database 2 } \\
\hline Classifier & Accuracy & SPU \\
\hline MFS-CNN-MLP & $96.2 \%$ & 17.2 \\
\hline MFS-CNN-SVM & $98.2 \%$ & 16.8 \\
\hline VGG-16 & $\mathbf{9 9 . 7 \%}$ & - \\
\hline
\end{tabular}

Table 6. Speedup Performance on Database 1 and 2 for Scenario 3, best results highlighted in bold.

\begin{tabular}{|c|c|c|}
\hline \multicolumn{3}{|c|}{ Database 1 } \\
\hline Classifier & Accuracy & SPU \\
\hline MFS-CNN-MLP & $96.4 \%$ & 16.9 \\
\hline MFS-CNN-SVM & $86.6 \%$ & 16.5 \\
\hline VGG-16 & $\mathbf{9 8 . 8 \%}$ & - \\
\hline \multicolumn{3}{|c|}{ Database 2 } \\
\hline Classifier & Accuracy & SPU \\
\hline MFS-CNN-MLP & $98.4 \%$ & 16.7 \\
\hline MFS-CNN-SVM & $96.6 \%$ & 16.6 \\
\hline VGG-16 & $\mathbf{9 9 . 7 \%}$ & - \\
\hline
\end{tabular}

Table 7. Speedup Performance on Database 1 and 2 for Scenario 4, best results highlighted in bold.

\begin{tabular}{|c|c|c|}
\hline \multicolumn{3}{|c|}{ Database 1 } \\
\hline Classifier & Accuracy & SPU \\
\hline MFS-CNN-MLP & $96.9 \%$ & 16.9 \\
\hline MFS-CNN-SVM & $88.8 \%$ & 16.5 \\
\hline VGG-16 & $\mathbf{9 8 . 8 \%}$ & - \\
\hline \multicolumn{3}{|c|}{ Database 2 } \\
\hline Classifier & Accuracy & SPU \\
\hline MFS-CNN-MLP & $98.7 \%$ & 16.7 \\
\hline MFS-CNN-SVM & $97.7 \%$ & 16.8 \\
\hline VGG-16 & $\mathbf{9 9 . 7 \%}$ & - \\
\hline
\end{tabular}


Table 8. Speedup Performance on Database 1 and 2 for Scenario 5, best results highlighted in bold.

\begin{tabular}{|c|c|c|}
\hline \multicolumn{3}{|c|}{ Database 1 } \\
\hline Classifier & Accuracy & SPU \\
\hline MFS-CNN-MLP & $95.0 \%$ & 17.2 \\
\hline MFS-CNN-SVM & $83.6 \%$ & 17.3 \\
\hline VGG-16 & $\mathbf{9 8 . 8 \%}$ & - \\
\hline \multicolumn{3}{|c|}{ Database 2 } \\
\hline Classifier & Accuracy & SPU \\
\hline MFS-CNN-MLP & $95.8 \%$ & 17.1 \\
\hline MFS-CNN-SVM & $95.8 \%$ & 17.4 \\
\hline VGG-16 & $\mathbf{9 9 . 7 \%}$ & - \\
\hline
\end{tabular}

Table 9. Speedup Performance on Database 1 and 2 for Scenario 6, best results highlighted in bold.

\begin{tabular}{|c|c|c|}
\hline \multicolumn{3}{|c|}{ Database 1 } \\
\hline Classifier & Accuracy & SPU \\
\hline MFS-CNN-MLP & $95.2 \%$ & 17.1 \\
\hline MFS-CNN-SVM & $83.2 \%$ & 17.0 \\
\hline VGG-16 & $\mathbf{9 8 . 8 \%}$ & - \\
\hline \multicolumn{3}{|c|}{ Database 2 } \\
\hline Classifier & Accuracy & SPU \\
\hline MFS-CNN-MLP & $96.1 \%$ & 17.0 \\
\hline MFS-CNN-SVM & $95.5 \%$ & 17.3 \\
\hline VGG-16 & $\mathbf{9 9 . 7 \%}$ & - \\
\hline
\end{tabular}

The VGG-16 model with 10 classes uses approximately 1.6 GB of RAM. The expansion of memory occupancy occurs during the training process. When the database has many images, the training process on computers with limited memory space without GPU turns the process impractical.

The memory occupied by the proposed method occurs mainly due to the tensor of maps stored in the memory, with $H \times H \times M \times K_{l}$ bytes per layer, where $H$ represents the dimensions of maps. We observed that the growth of memory occupation is dependent on the number of $M$ samples. The value of $M$ needs to be great when the database has a large number of samples and classes. This rise is due to the necessity of representation of the total diversity of the database. Due to this drawback, the extraction of matrices of eigenvectors and mean vectors is infeasible when the image database has a large number of samples.

When the classification process of a new sample occurs in the VGG-16 model, the occupation of memory is due mainly to the storage of part of kernels weighs and the creation of the $K l$ feature maps in the current layer in forwarding propagation mode. In the classification task, the proposed method occupies memories mainly with matrices of eigenvectors and mean vectors. The size of low dimensional samples is only of few kilobytes.

To perform the experiments, we used the Google Colab service. The service offers a cloud computing server with $32 \mathrm{~GB}$ of RAM and an Nvidia Tesla K80 GPU, Nvidia Tesla T10 or similar. The service was used only to train the original VGG-16 model. To extract the feature maps, compute the eigenvectors, train the external classifiers, and execute the test experiments, it was used a personal computer with $8 \mathrm{~GB}$ of RAM and an Intel Core i5 Vpro processor.

All processes for extracting and storage the matrices of eigenvectors and mean vectors lasted six hours. The size of archives totalled $685 \mathrm{MB}$ of RAM for 10 classes. The proposed method 
achieved satisfactory results in the experiments but demonstrates be not feasible with many classes and samples. This drawback is due to the high occupancy memory by the tensors.

Additionally, the method is not effective when the objects of interest in the images have a high variance of size and position and not aligned since the method is based on linear PCA.

In recent years, different works achieved good results by pruning [22] or compressing [23] large $\mathrm{CNN}$ architectures. However, our approach uses the ranked eigenvectors for reducing the classification time and not reducing the size of the architecture.

\section{Conclusions}

In this paper, we presented an alternative method that focuses on the knowledge's of CNN's kernels associated with a low complexity classifier to reduce the time of classification while preserving part of the performance reached by CNN.

The results have shown that MFS-CNN is efficient in ADAS classification problems with a limited number of classes. The method is helpful in classification applications that use CNNs for embedded applications, with low computational resources in computer vision applications for the autonomous vehicle. The experiments with scenario 4 and 6 showed a reasonable accuracy with a high speed-up rate. In scenarios 4 and 6, we randomized the eigenvectors selection and, even though the loss in accuracy was minimal. It is an indication that we have a high redundancy spread along all eigenvectors.

In the next step of this research, we will extend the application for other ADAS problems, such as license plate and vehicle type classification. The low consumption of the method turns the implementation and operation appropriate to the vehicular low-cost embedded platforms. These platforms are used mainly for performing real-time computer vision tasks.

In addition, we will evaluate a method to choose the minimum amount of the most significant eigenvectors, not considering only the eigenvalues as mentioned in this work, but the accuracy and reduced time for classification. The new version of MFS-CNN will handle reasonably a high number of samples and classes, outperforming the current drawback.

\section{REFERENCES}

[1] YannLeCun and YoshuaBengio, (1998) "Convolutional networks forimages, speech, and time series", The handbook of brain theory and neural networks. MIT Press, Cambridge, MA, USA, 255258.

[2] Y. Lecun, L. Bottou, Y. Bengio and P. Haffner , (1998) "Gradient-based learning applied to document recognition", Proceedings of the IEEE, vol. 86, no. 11, pp. 2278-2324.

[3] H. R. Kher and V. K. Thakar, (2014) "Scale Invariant Feature Transform Based Image Matching and Registration", Fifth International Conference on Signal and Image Processing, Bangalore, India, pp. 50-55.

[4] N. Dalal and B. Triggs, (2005) "Histograms of oriented gradients for human detection", IEEE Computer Society conference on Computer Vision and Pattern Recognition (CVPR'05), San Diego, CA, USA, 2005, pp. 886-893.

[5] Alex Krizhevsky, IlyaSutskever, and Geoffrey E. Hinton, (2012) "ImageNet classification with deep convolutional neural networks", In Proceedings of the 25th International Conference on Neural Information Processing Systems - Volume 1 (NIPS'12), Curran Associates Inc., Red Hook, NY, USA, $1097-1105$.

[6] K.Simonyan and A. Zisserman, (2014) "Very deep convolutional networks for large-scale image recognition", in International Conference on Learning Representation (ICLR),San Diego, CA, USA. 
[7] Sandler, M. et al, (2018) "Mobilenetv2: Inverted residuals and linear bottlenecks", in proceedings of the IEEE conference on computer vision and pattern recognition. [S.1.: s.n.], p. 4510-4520.

[8] Huang, R.; Pedoeem, J.; Chen, C., (2018) "Yolo-lite: A real-time object detection algorithm optimized for non-gpu computers", In: . [S.1.: s.n.], p. 2503-2510.

[9] X. Zhang, J. Zou, K. He and J. Sun., (2016) "Accelerating Very Deep Convolutional Networks for Classification and Detection", Pattern Analysis and Machine Intelligence, vol. 38, no. 10, pp. 19431955.

[10] V. Vanhoucke, A. Senior, and M. Z. Mao, (2011) "Improving the speed of neural networks on CPUs,"in Deep Learning and Unsupervised Feature Learning Workshop, NIPS.

[11] E. Denton, W. Zaremba, J. Bruna, Y. LeCun and R. Fergus, (2014) "Exploiting linear structure within convolutional networks for efficient evaluation", in Advances in Neural Information Processing Systems (NIPS).

[12] J. Huang et al., (2017) "Speed/Accuracy Trade-Offs for Modern Convolutional Object Detectors", 2017 IEEE Conference on Computer Vision and Pattern Recognition (CVPR), Honolulu, HI, 2017, pp. 3296-3297.

[13] M. Wang, B. Liu and H. Foroosh, (2017) "Factorized Convolutional Neural Networks", 2017 IEEE International Conference on Computer Vision Workshops (ICCVW), Venice, 2017, pp. 545-553.

[14] I. Garg, P. Panda and K. Roy, (2020) "A Low Effort Approach to Structured CNN Design Using PCA", in IEEE Access, vol. 8, pp. 1347-1360.

[15] J. M. Alvarez and M. Salzmann., (2017) "Compression-aware training of deep networks", CoRR, vol. abs/1711.02638.

[16] Ian Goodfellow, YoshuaBengio, and Aaron Courville., (2016) "Deep Learning”, The MIT Press.

[17] I.T Jolliffe, (2002) "Principal Components Analysis", 2nd Ed. Springer Series in Statistics.

[18] H. Lu, K. N. Plataniotis and A. N. Venetsanopoulos, (2008) "MPCA: Multilinear Principal Component Analysis of Tensor Objects", in IEEE Transactions on Neural Networks, vol. 19, no. 1, pp. 18-39.

[19] J. Arrospide, L. Salgado, M. Nieto, (2012) "Video analysis based vehicle detection and tracking using an MCMC sampling framework", EURASIP Journal on Advances in Signal Processing, vol. 2012.

[20] S. Houben, J. Stallkamp, J. Salmen, M. Schlipsing, C. Igel, (2013) "Detection of Traffic Signs in Real-World Images: The German Traffic Sign Detection Benchmark", International Joint conference on Neural Networks, 1288, 2013.

[21] Krizhevsky, Alex, (2012) "Learning Multiple Layers of Features from Tiny Images, University of Toronto, Sourced from Microsoft Academichttps://academic.microsoft.com/paper/3118608800.

[22] X. Ruan et al., (2020) "EDP: An Efficient Decomposition and Pruning Scheme for convolutional Neural Network compression", in IEEE Transactions on Neural Networks and Learning Systems.

[23] X. Yu, T. Liu, X. Wang and D. Tao, (2017) “On Compressing DeepModels by Low Rank and Sparse Decomposition", 2017 IEEE Conference on Computer Vision and Pattern Recognition (CVPR), Honolulu,HI, 2017, pp. 67-76.

\section{AUTHORS}

Michel André L. Vinagreiro Is graduate in engineering, master's student and researcher with Laboratory of Integrated systems, Escola Politecnica da Universidade de Sao Paulo, Sao Paulo, Sao Paulo, Brasil.

Edson C. Kitani Is P.h.D degree by Escola Politecnica da Universidade de Sao Paulo andgraduation professor in Fatec Santo Andre, Santo Andre, São Paulo, Brasil.
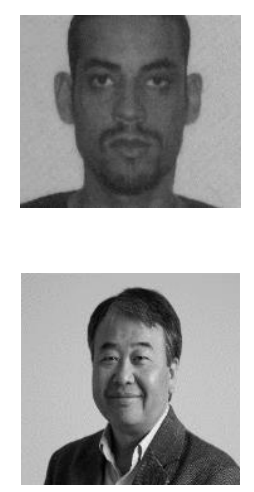
Armando AntonioM. Lagana Is P.h.Ddegreeby Escola Politecnica da Universidade de Sao Paulo, graduation professor and researcher with Laboratory of Integrated systems, Escola Politecnica da Universidade de Sao Paulo, Sao Paulo, Sao Paulo, Brasil.

Leopoldo R. Yoshioka Is P.h.D degree by Tokyo Institute of Technology, graduation professor and researcher with Laboratory of Integrated systems, Escola Politecnica da Universidade de Sao Paulo, Sao Paulo, Sao Paulo, Brasil.
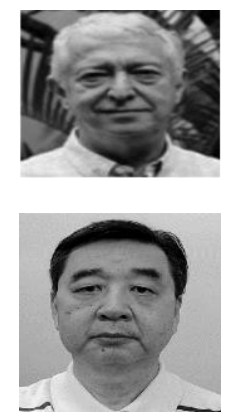

(C) 2021 By AIRCC Publishing Corporation. This article is published under the Creative Commons Attribution (CC BY) license. 